\title{
CORRELATION AND GENETIC ANALYSES OF DIFFERENT CHARACTERISTICS IN SAUDI ARABIAN WHEAT REVEAL CORRELATION NETWORKS AND SEVERAL TRAIT-ASSOCIATED MARKERS
}

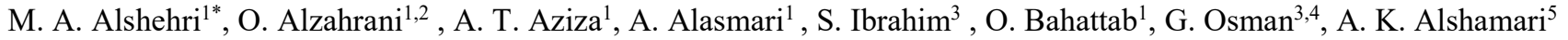 \\ and S. A Alduaydi ${ }^{5}$ \\ ${ }^{1}$ Biology Department, Faculty of Science, University of Tabuk, Tabuk, Saudi Arabia \\ ${ }^{2}$ Genome and Biotechnology Unit, Faculty of Sciences, University of Tabuk, Tabuk, Saudi Arabia. ${ }^{3}$ Agricultural Genetic \\ Engineering Research Institute (AGERI), Agriculture Research Center (ARC), Giza, Egypt \\ ${ }^{4}$ Department of Biology, Faculty of Applied Science, Umm Al-Qura University, Makkah, Saudi Arabia. \\ ${ }^{5}$ Plant Gene Bank, National Agriculture and Animal Resources Research Center, Ministry of Environment water and \\ Agriculture, Saudi Arabia \\ *Corresponding author's E-mail: ma.alshehri@ut,edu.sa
}

\begin{abstract}
Thirty-six different morpho-agronomic traits in 40 different accessions of Saudi Arabian wheat were investigated through marker-trait association analysis, which could provide a single PCR marker. A total of 25 different correlations were retrieved, among which, days to heading (DH) was negatively correlated with flag leaf anthocyanin (FLA) ( $\mathrm{r}=-$ $0.73)$ and positively correlated with plant height $(\mathrm{PH})(\mathrm{r}=0.63)$ and leaf length $(\mathrm{LL})(\mathrm{r}=0.62)$. Additionally, seeds count on spike (SCS) was positively correlated with awn color (AC) $(r=0.67)$ and spike color $(\mathrm{SC})(\mathrm{r}=0.61)$ and negatively correlated with grain shape (GS) $(r=-0.6)$. Additionally, 19 PCR primers belonging to three different types of markers (ISSR, SSR, and SCoT) were used to study the population structure and diversity and to investigate the association between different agronomic traits. The total number of bands (TNB) produced by different molecular marker assays was 158. The number of polymorphic bands (PB) ranged from 1 (SCoT3 and SSR14) to 10 (SCoT5, and SCoT35), with a mean of 4.7 bands per primer. The polymorphism percentage (PP) for primers ranged from $14 \%$ (SCoT3) to $100 \%$ (SSR9, SSR10, and SSR2), with an average of $60 \%$ per primer. Thirty-seven molecular markers (7 SSRs, 26 SCoTs, and 4 ISSRs) manifested significant associations with 29 wheat plant traits. Some markers were associated with more than one agronomic trait. These findings could support Saudi Arabian wheat breeding programs by providing several markers associated with agronomic traits that could be used in marker-assisted selection in local wheat accessions.
\end{abstract}

Keywords: Wheat, SSR, SCoT, ISSR, Saudi wheat, traits correlation, marker-assisted selection.

https://doi.org/10.36899/JAPS.2020.6.0169

Published online August 03,2020

\section{INTRODUCTION}

Wheat is a fundamental nutritional source of calories and proteins for a variety of human populations, which makes it a strategic crop and one of the most important cereal crops (Curtis et al., 2002). Global wheat production exceeded 700 million tonnes in 2015/2016, valued over 145 billion dollars per year (Figueroa et al., 2018). With distinctive characteristics of cultivars and different end-use quality traits, wheat is adapted to divergent production environments and can fulfill different types of human needs. Additionally, wheat is a major industrial crop and a source of raw material in feed mills, livestock feeds, and human consumptions (Westendorf, 2000). Grain yield is a complicated trait which is mainly controlled by several yield component traits such as spikes per unit area, grains per spike and grain weight. It seems, however, that heavier grains have less chance for genetic improvement in wheat yield
(Fischer, 2011). In order to design a well-constructed breeding program by implementing selection strategies for grain yield, important pieces of information regarding the diversity of cultivars and the association between grain yield components must be retrieved. Correlation studies of agronomic traits give a better view toward comprehending the association of different traits with grain yield that could be of great help to wheat breeders (Muhammad et al., 2011). Moreover, wheat hypersensitivity to pathogens, pests, and high temperature affect its sustainability, cultivation, and production in areas where it is continuously threatened by environmental changes (Muhammad et al., 2011; Skoracka et al., 2018). Molecular markers are an irreplaceable tool for genetic improvement of crops and they could be used to investigate the genetic diversity and population structure in the studied genotypes. Additionally, they are efficient tools for marker-assisted selection as well as saving labor, time and financial resources in breeding programs (Ibrahim et al., 2016). 
Marker-trait association analysis is based on the statistical connection between the specific PCR marker and the observed phenotype. It measures the redundancy of co-occurrence of the observed phenotype in a population, which is nonrandom and more than expected (Munshi and Osman, 2010). This association analysis plays an important part in population genetics in understanding the genetic etiology of complex diseases and traits (Lowe et al., 2015). Successful application of this analysis in salt- (Munir et al., 2013) and drought(Quarrie et al., 2003) tolerant and disease-resistant crops (Qi et al., 2008) has confirmed its incomparable advantages. Advanced molecular marker analysis could be used to investigate the relationship between genes and traits and the correlation between PCR-based molecular markers and gene networks in plants, thus giving crop breeders more control over plant genetic resources with less effort (Alsamman et al., 2019; Habib et al., 2019). Start codon targeted (SCoT) assay targets the start codon (ATG) and its primer design reduces the efficiency of unwanted and random amplification of genomic regions. Both annealing temperature and primer length do not affect the reproducibility of dominant markers like SCoT (Collard and Mackill, 2009).

The integration of SCoT molecular markers in breeding different plant species such as coconut (Rajesh et al., 2015), jojoba (Heikrujam et al., 2015), tomato (Abdein et al., 2018) and olive (Alsamman et al., 2017) has been reported. Inter-simple sequence repeat (ISSR) markers are used to target nucleotide repeats in regions of plant genome containing simple sequence repeats (SSRs). ISSR technique has high repeatability and polymorphism and is suitable for studying intra and inter populational genetic variability in different plant species. Additionally, it has been used to study the genetic diversity in plant species such as barley (Fernandez et al., 2002), durum wheat (Alireza et al., 2016), tomato and garlic (Abdein et $a l ., 2018$ ). The aim of the current paper is to identify those traits which most strongly influence the variation in final yield and the correlation networks of different agronomic traits by means of the analysis of traits in wheat. Furthermore, the identification of PCR markers which are linked to the observed phenotypic variation and could be used for marker-assisted selection provides a better understanding of the genetic structure of wheat plant traits. Moreover, the present study investigates whether the genetic diversity in wheat can be partly explained by ISSR, SSR and SCoT polymorphic markers in order to establish the relationships between different wheat genotypes.

\section{MATERIALS AND METHODS}

Plant material: Forty wheat genotypes were collected from the Saudi Arabia region. A total of 36 different morpho-agronomic traits measured were as follows: awn color (AC), awn direction of ear (AD), awn length (AL), awn presence (AP), anthocyanin stain (AS), cross section thickness of the plant leg (CSPL), day to heading (DH), flag leaf anthocyanin (FLA), flag leaf bud (FLB), flag leaf hair (FLH), flag leaf length (FLL), flag leaf width (FLW), grain color (GC), growth rate (GR), grain shape (GS), grain wrinkle $(\mathrm{GW})$, hair density on glume (HDG), leaf color (LC), length of glume lower peak (LGLP), leg length (LL), one thousand seed weight (OTSW), plant height (PH), spike color (SC), seed count on spike (SCS), shape of glume (SG), shape of glume lower peak (SGLP), shape of glume shoulder (SGS), seeds hair (SH), spike length (SL), seeds phenolic color degree (SPCD), spike shape (SS), wax layer of flag leaf (WLFL), wax layer of flag leaf blade (WLFLB), wax layer of leg neck (WLLN), wax layer on the spike (WLS), and wheat seasonal mode (WSM) (Table 1).

DNA extraction and PCR-based molecular marker analysis: DNeasy Plant Mini Kit (Qiagen, New York, NY, USA) was used to extract total DNA. DNA quality and quantity were estimated using gel electrophoresis and DNA samples were stored at $-20{ }^{\circ} \mathrm{C}$. A total of two ISSR, twelve SCoT and five SSR primers were used in this study (Table 2). The PCR amplifications were performed in reactions with templates having GC content during the PCR cycles according to Ibrahim et al. (2016) and Ahla et al. (2019). The final PCR products were stored at $4{ }^{\circ} \mathrm{C}$. The ethidium bromide-stained agarose gel $(1.5 \%)$, which was used to separate DNA fragments, was documented using the Gel Doc XR system (Bio-Rad, Hercules, CA, USA).

Statistical and genetic analyses: Correlation analysis between agronomic and morphological traits was performed using the R package "Hmisc" (Harrell and Harrell, 2019) and the correlation coefficients (r) were estimated using the "corrplot" (Taiyun et al., 2017). The marker-trait association analysis based on F-test statistics was conducted using PowerMarker software (Liu and Muse, 2005). Correlations and associations were considered statistically significant if the p-value was lower than $0.05(\mathrm{P}<0.05)$. In order to identify the markers linked with different agronomic traits, an online web tool called ClustVis was used to visualize a heat map from the similarity matrix (Metsalu and Vilo, 2015). PCR fragments were scored as present (1) or absent (0). Dice similarity coefficients between different samples were calculated using the unweighted pair group method with arithmetic averages (UPGMA) and they were used for the construction of phylogenetic tree or dendrogram using the Paste software. The population was studied using the STRUCTURE software with burn-in and 100,000 MCMC iterations (Pritchard et al., 2000). The results were assessed using STRUCTURE HARVESTER to determine the number of populations (Earl and others, 2012). 


\section{RESULTS AND DISCUSSION}

Correlation analysis of morpho-agronomic traits: Grain production is a complex phenomenon, involving many contributing parameters, which control grain production, both indirectly and directly. Breeders are naturally interested in exploring the size and type of association between the morpho-agronomic traits (Saima et al., 2018). Correlations among plant traits mainly indicate potential trade-offs or allometric relationships in biological processes like carbon gain, support, water uptake, and reproduction that are related to different plant organs (Saima et al., 2018). In this study, the correlations among 36 different morpho-agronomic traits in wheat were investigated. Among a total of 25 different correlations that were retrieved, the maximum positive correlation was between PH and LL $(r=0.92)$, while the maximum negative correlation was observed between GS and GW ( $\mathrm{r}=-0.93)$. Days to heading (DH) was negatively correlated with flag leaf anthocyanin (FLA) (r $=-0.73)$ and positively correlated with plant height $(\mathrm{PH})$ $(\mathrm{r}=0.63)$ and leaf length $(\mathrm{LL})(\mathrm{r}=0.62)$. Additionally, seed counts on spike (SCS) showed positive correlation with awn color $(\mathrm{AC})(\mathrm{r}=0.67)$ and spike color $(\mathrm{SC})(\mathrm{r}=$ 0.61 ), whereas it was negatively correlated with grain shape (GS) ( $\mathrm{r}=-0.6)$ (Table 3, Fig. 1). The performance, correlation, and cluster analysis for various quantitative traits including grain yield among the wheat material exotic to Pakistan showed that days to heading (DH) had significant positive correlations with days to maturity $(\mathrm{r}=$ $0.7995)$, spikelets per spike $(\mathrm{r}=0.4391)$, plant height $(\mathrm{r}=$ 0.3168), and spike length $(\mathrm{r}=0.2696)$ (Saima et al., 2018). Moreover, the study on the heritability and variance components of root traits in wheat under drought stress revealed that the highest correlation between days to heading and grain yield was observed under nonstressed conditions ( $\mathrm{r}=0.72)$ (Alsamman et al., 2017). Figure 2 shows the correlation coefficients (r) higher than 0.5 among studied traits. The results of correlation network analysis demonstrated the correlations between GS, SH, GW, SC, AC, SS, SCS, and HGD and between $\mathrm{PH}, \mathrm{LL}, \mathrm{DH}$, and FLA. Furthermore, it showed that the changes in some traits of wheat would affect most traits directly or indirectly.

Molecular marker analysis: All 19 PCR primers produced scorable bands (Figure 3 and Table 3 ). The total number of bands (TNB) produced by different molecular marker assays was 158 . The total number of bands ranged from 2 (SSR14, SSR9, and SSR10) to 14 (SCoT16), with a mean of 8.3 bands per primer. The number of polymorphic bands (PB) varied between 1 (SCoT3 and SSR14) and 10 (SCoT5 and SCoT35) with an average of 4.7 bands per primer. The polymorphism percentage (PP) ranged from 14\% (SCoT3) to 100\% (SSR9, SSR10, and SSR2), with a mean of $60 \%$ per primer. The minor allele frequency (MAF) ranged from 0.383 (SCoT13 and SCoT35) to 0.85 (SSR14 and SSR10) with a mean of 0.383 per primer. The polymorphism information content (PIC) ranged from 0.22 (SSR14) to 1 (SCoT13). In order to study the genetic diversity in some durum wheat genotypes using six SCoT primers (Alireza et al., 2016), 54 polymorphic bands with $100 \%$ PP were obtained. SCoT markers whose moderate potential to detect genetic variation compared to other molecular markers has been reported, were used for detecting allelic variation among different olive genotypes (Alsamman et al., 2017). Moreover, to examine the genetic diversity and population structure of several Jatropha curcas L. genotypes using the ISSR assay, 11 ISSR primers were tested and they generated a total number of 307 bands (TNB) and 294 polymorphic bands (PB) (Gomes et al., 2018). In barley, the PIC mean value was 0.636 , and it ranged from 0.351 to 0.874 (Yong-Cui et al., 2005), which confirmed the results of the present study on the high efficiency of ISSR markers in exhibiting high PIC values.

Population structure and genetic diversity: Population structure analysis involves assigning each individual in a population to a cluster and then reporting the number of clusters. This analysis has many applications in genetic studies including grouping individuals, identifying immigrants and inferring the demographic history of populations. There are several approaches to population structure inference, such as principal component analysis (PCA), detrended correspondence analysis (DCA) and allele frequency-based analyses (Lee et al., 2009).

Detrended correspondence analysis (DCA) is a multivariate statistical method mainly used by researchers to identify the main factors in large and sparse data matrices that represent the structure of ecological community data (Hill and Gauch, 1980). Figure 4 demonstrates the population structure based on detrended correspondence analysis (DCA). A total of eight accessions including 23, 24, 25, 26, 13, 15, 16, and 36 were separated from the other wheat accessions, forming two separate groups (clusters). Accession 37 was somewhat different from most of the accessions. Principal component analysis (PCA) is a method for reducing the dimension, which uses an orthogonal transformation in exploratory data analysis to visualize genetic distances and relatedness between individuals belonging to different populations (Lee et al., 2009). PCA analysis confirmed the predication about the population structure of the wheat accessions based on the detrended correspondence analysis (DCA) (Fig. 5). Additionally, the population structures of two clusters became more condensed and the eight accessions formed one population separated from the other accessions. Population structure analysis by STRUCTURE software is commonly used to infer population structure and to 
assign individuals to different clusters given the allele frequencies using Markov Chain Monte Carlo (MCMC) (Pritchard et al., 2000). Population structure inference using STRUCTURE software confirmed results obtained using population structure analysis. A set of wheat accessions was divided into two different populations, where $23,24,25,26,13,15,16$ and 36 belonged to one population with $85 \%$ similarity and the other accessions were clustered into another population. Additionally, the population containing accession 37 with a significantly high score was separated from the others (Fig. 6). The studies of genetic diversity based on the molecular markers were conducted and data, which were transformed into binary form, were used for phylogenetic analysis. Accession 31 with the similarity of $86 \%$ was separated from the main group (Figure 7).

Marker-trait association analysis: Statistical analysis of breeding systems coupled with genetic data is an efficient method for the use of a variety of crop species in professional breeding programs (Danail et al., 2010). In this study, SSR, SCoT, and ISSR assays produced markers associated with several traits in wheat. Only markers with association scores higher than $0.05\left(-\log _{10}\right.$ $(p$-value $)=1.3)$ were considered. A total of 37 molecular markers (7 SSRs, 26 SCoTs, and 4 ISSRs) manifested significant associations with 29 traits in wheat. Association of marker SCoT3 ${ }_{170}$ (marker band size) with PH had the highest significance score $\left(-\log _{10}(\mathrm{p}\right.$-value $)=$
6.9). Additionally, several markers were associated with more than one trait. The SCoT33 650 was linked to SCS, SL, AC, HDG, GS, GW, and FLB. However, the SCoT16 $6_{580}$ was linked to FLL, PL, SGLP, LL, and FLA and the SCoT2 600 was associated with AC, HDG, GS, $\mathrm{GW}$, and FLA. These markers could be used to target more than one trait and to follow the trait-trait correlation network (Fig. 8, Table 4). Marker-trait association analysis was used to identify SSR markers associated with salt tolerance in chickpea genotypes (Shaimaa et al., 2019). By using SSR markers, 40 PCR-based markers associated with 13 different agronomic traits have been identified and reported in Lotus japonicus, which could explain the phenotypic variation in stem color from genotypes (Gondo et al., 2007). Moreover, 14 traits related to salt-tolerance and 46 trait-associated markers were detected in barley (Elakhdar et al., 2016).

Association analysis of different traits in wheat was conducted based on shared trait-associated molecular markers. Figure 9 shows that some traits in wheat formed linked groups, where several traits were clustered into one block with a large number of shared markers. Traits such as SCS, SL, FLB, AC, HDG, DH, AL, GW and GS were grouped into one block, while WLLN, SS, WLS, and LC were clustered into another. Furthermore, GR, FLA, SGLP, FLL, PL, and LL were divided into one cluster, whereas the cluster containing PH and LL had the highest number of shared markers.

Table 1. Plant number (PN), local name (LN) and region (Reg.) for the forty wheat accessions used in this study.

\begin{tabular}{|c|c|c|c|c|c|}
\hline PN & $\mathbf{L N}$ & Reg. & PN & $\mathbf{L N}$ & Reg. \\
\hline 1 & Helba & Barida & 21 & Baladi & Koara-Elkaseem \\
\hline 2 & Maiaa & Barida & 22 & Maiaa Baladi & Taimaa-Tabok \\
\hline 3 & Lokami & Barida & 23 & Lokami & Koara-Elkaseem \\
\hline 4 & Samaa & Tamir & 24 & Unknown & Abhaa-Aseer \\
\hline 5 & Soariak & Tamir & 25 & Unknown & Aseer \\
\hline 6 & Samaa Baladi & Tamir & 26 & Shokia & Elbaha \\
\hline 7 & Lokami Abiad & Tamir & 27 & Unknown & Tabok \\
\hline 8 & Henta Asmr & Tamir & 28 & Unknown & Tabok \\
\hline 9 & Baal & Tamir & 29 & Unknown & Tabok \\
\hline 10 & Bor Baladi & Aldalim & 30 & Bor Samraa & Najran \\
\hline 11 & Molloaha Mokaom & Alehsaa & 31 & Bor Zarai & Najran \\
\hline 12 & Samaa Baladi & Elkharag & 32 & Bor Henta & Elkaseem \\
\hline 13 & Asmr & Najran & 33 & Hab Bar Atrali & Aseer \\
\hline 14 & Somiraa & Elnamas & 34 & Hab Bar Saib & Aseer \\
\hline 15 & Kias & Belsamr-Aseer & 35 & Unknown & Najran \\
\hline 16 & Saib & Elnamas & 36 & Lokami & Khobraa-Elkaseem \\
\hline 17 & Maiaa Baladi & Elnamas & 37 & Bor & Khobraa-Elkaseem \\
\hline 18 & Khobraa-Elkaseem & Khobraa-Elkaseem & 38 & Kawara & Barida \\
\hline 19 & Maiaa & Elbakiria & 39 & Morgan & Barida \\
\hline 20 & Ymani Baladi & Tabok & 40 & Kasim & Barida \\
\hline
\end{tabular}


Table 2. Primers name (PN), Sequences were used in this study.

\begin{tabular}{|c|c|c|c|}
\hline PN & Sequence & PN & Sequence \\
\hline ISSR-14 & 5'-СТССТССТССТССТСТТ-3' & SCoT-2 & 5'-CAACAATGGCTACCACCC-3' \\
\hline ISSR-15 & 5'-СТСТСТСТСТСТСТCTRG-3' & SCoT-3 & 5'-CAACAATGGCTACCACCG-3' \\
\hline \multirow[t]{2}{*}{ SSR-2 } & F. 5' TCATTGGTAATGAGGAGAGA-3' & SCoT-4 & 5'-CAACAATGGCTACCACCT-3' \\
\hline & R. 5' GAACCATTCATGTGCATGTC-3' & SCoT-5 & 5'-CAACAATGGCTACCACGA-3' \\
\hline \multirow[t]{2}{*}{ SSR-9 } & F. 5' AATTTCAAAAAGGAGAGAGA-3' & SCoT-11 & 5'-AAGCAATGGCTACCACCA-3' \\
\hline & R. 5' AACATGTGTTTTTAGCTATC-3' & SCoT-12 & 5'-ACGACATGGCGACCAACG-3' \\
\hline \multirow[t]{2}{*}{ SSR-10 } & F. 5' AAGATGGACGTATGCATCACA-3' & SCoT-13 & 5'-ACGACATGGCGACCATCG-3' \\
\hline & R. 5'GCCATATTTGATGACGCATA-3' & SCoT-14 & 5'-ACGACATGGCGACCACGC-3' \\
\hline \multirow[t]{2}{*}{ SSR-14 } & F. 5' CGACCCGGTTCACTTCAG 3' & SCoT-16 & 5'-ACCATGGCTACCACCGAC-3' \\
\hline & R. 5' AGTCGCCGTTGTATAGTGCC 3' & SCoT-20 & 5'-ACCATGGCTACCACCGCG-3' \\
\hline \multirow[t]{2}{*}{ SSR-16 } & F.5 GCGGGTCGTTTCCTGGAAATTCATCTAA 3' & SCoT-33 & 5'-CATGGCTACCACCGGCCC-3' \\
\hline & R. 5' GCGAAATGATTGGCGTTACACCTGTTG 3 & SCoT-35 & 5'-CCATGGCTACCACCGCAG-3' \\
\hline
\end{tabular}

Table 3 The significant correlation scores (r) between different wheat traits.

\begin{tabular}{|c|c|c|c|c|c|c|c|c|}
\hline Trait & Trait & $\mathbf{R}$ & Trait & Trait & $\mathbf{R}$ & Trait & Trait & $\mathbf{r}$ \\
\hline SG & SGS & $0.63^{*}$ & SCS & $\mathrm{AC}$ & $0.67^{* *}$ & GS & SS & $-0.67^{* *}$ \\
\hline \multirow{2}{*}{ LL } & DH & $0.62^{*}$ & & $\mathrm{SC}$ & $0.61^{*}$ & & SCS & $-0.6^{*}$ \\
\hline & PL & $0.92^{* * *}$ & & GS & $-0.6^{*}$ & & $\mathrm{AC}$ & $-0.84^{* * *}$ \\
\hline \multirow[t]{3}{*}{ PL } & FLA & $-0.61^{*}$ & HDG & $\mathrm{AC}$ & $0.82^{* * *}$ & & HDG & $-0.68^{* *}$ \\
\hline & $\mathrm{DH}$ & $0.63^{*}$ & & GS & $-0.68^{* *}$ & & $\mathrm{SC}$ & $-0.9^{* * *}$ \\
\hline & LL & $0.92^{* * *}$ & & GW & $0.63^{*}$ & & GW & $-0.93^{* * *}$ \\
\hline \multirow[t]{5}{*}{ GW } & SS & $0.62^{*}$ & SGS & SG & $0.63^{*}$ & $\mathrm{AL}$ & AP & $0.6^{*}$ \\
\hline & $\mathrm{AC}$ & $0.79^{* * *}$ & $\mathrm{AC}$ & SCS & $0.67^{* *}$ & $\mathrm{SH}$ & $\mathrm{SC}$ & $-0.6^{*}$ \\
\hline & HDG & $0.63^{*}$ & & HDG & $0.82^{* * *}$ & & & \\
\hline & $\mathrm{SC}$ & $0.83^{* * *}$ & & $\mathrm{SC}$ & $0.73^{* * *}$ & & & \\
\hline & GS & $-0.93^{* * *}$ & & GS & $-0.84^{* * *}$ & & & \\
\hline WLFLB & WLLN & $0.61^{*}$ & & GW & $0.79^{* * *}$ & & & \\
\hline \multirow[t]{3}{*}{ SS } & $\mathrm{SC}$ & $0.75^{* * *}$ & DH & FLA & $-0.73^{* * *}$ & & & \\
\hline & GS & $-0.67^{* *}$ & & PL & $0.63^{*}$ & & & \\
\hline & GW & $0.62^{*}$ & & LL & $0.62^{*}$ & & & \\
\hline \multirow[t]{6}{*}{$\mathrm{SC}$} & SS & $0.75^{* * *}$ & AP & $\mathrm{AL}$ & $0.6^{*}$ & & & \\
\hline & SCS & $0.61^{*}$ & WLS & WLLN & $0.67^{* *}$ & & & \\
\hline & $\mathrm{AC}$ & $0.73^{* * *}$ & FLA & DH & $-0.73^{* * *}$ & & & \\
\hline & GS & $-0.9^{* * *}$ & & PL & $-0.61^{*}$ & & & \\
\hline & GW & $0.83^{* * *}$ & WLLN & WLFLB & $0.61^{*}$ & & & \\
\hline & $\mathrm{SH}$ & $-0.6^{*}$ & & WLS & $0.67^{* *}$ & & & \\
\hline
\end{tabular}

${ }^{*}$ significant

Table 4. primer name (PN), total number of PCR bands (TB), monomorphic bands (MB), polymorphic bands (PB), marker allele frequency (MAF) and polymorphism information content (PIC).

\begin{tabular}{ccccccc}
\hline PN & TB & MB & PB & PP & MAF & PIC \\
\hline ISSR14 & 8 & 5 & 3 & 0.38 & 0.55 & 0.62 \\
ISSR15 & 9 & 3 & 6 & 0.67 & 0.28 & 0.87 \\
SCoT11 & 11 & 5 & 6 & 0.55 & 0.2 & 0.88 \\
SCoT12 & 11 & 5 & 6 & 0.55 & 0.18 & 0.92 \\
SCoT13 & 11 & 2 & 9 & 0.82 & 0.08 & 0.96 \\
SCoT14 & 13 & 9 & 4 & 0.31 & 0.55 & 0.65 \\
SCoT16 & 14 & 8 & 6 & 0.43 & 0.23 & 0.86 \\
SCoT2 & 11 & 5 & 6 & 0.55 & 0.18 & 0.89 \\
SCoT20 & 11 & 6 & 5 & 0.45 & 0.33 & 0.82 \\
\hline
\end{tabular}




\begin{tabular}{ccccccc}
\hline SCoT3 & 7 & 6 & 1 & 0.14 & 0.7 & 0.33 \\
SCoT33 & 7 & 3 & 4 & 0.57 & 0.18 & 0.89 \\
SCoT35 & 12 & 2 & 10 & 0.83 & 0.08 & 0.95 \\
SCoT4 & 6 & 3 & 3 & 0.5 & 0.43 & 0.63 \\
SCoT5 & 13 & 3 & 10 & 0.77 & 0.13 & 0.95 \\
SSR10 & 2 & 0 & 2 & 1 & 0.85 & 0.24 \\
SSR14 & 2 & 1 & 1 & 0.5 & 0.85 & 0.22 \\
SSR16 & 5 & 1 & 4 & 0.8 & 0.28 & 0.82 \\
SSR2 & 3 & 0 & 3 & 1 & 0.75 & 0.37 \\
SSR9 & 2 & 0 & 2 & 1 & 0.45 & 0.55 \\
Total & 158 & 67 & 91 & 11.8 & 7.28 & 13.4 \\
Mean & 8.3 & 3.5 & 4.7 & 0.62 & 0.38 & 0.70 \\
\hline
\end{tabular}

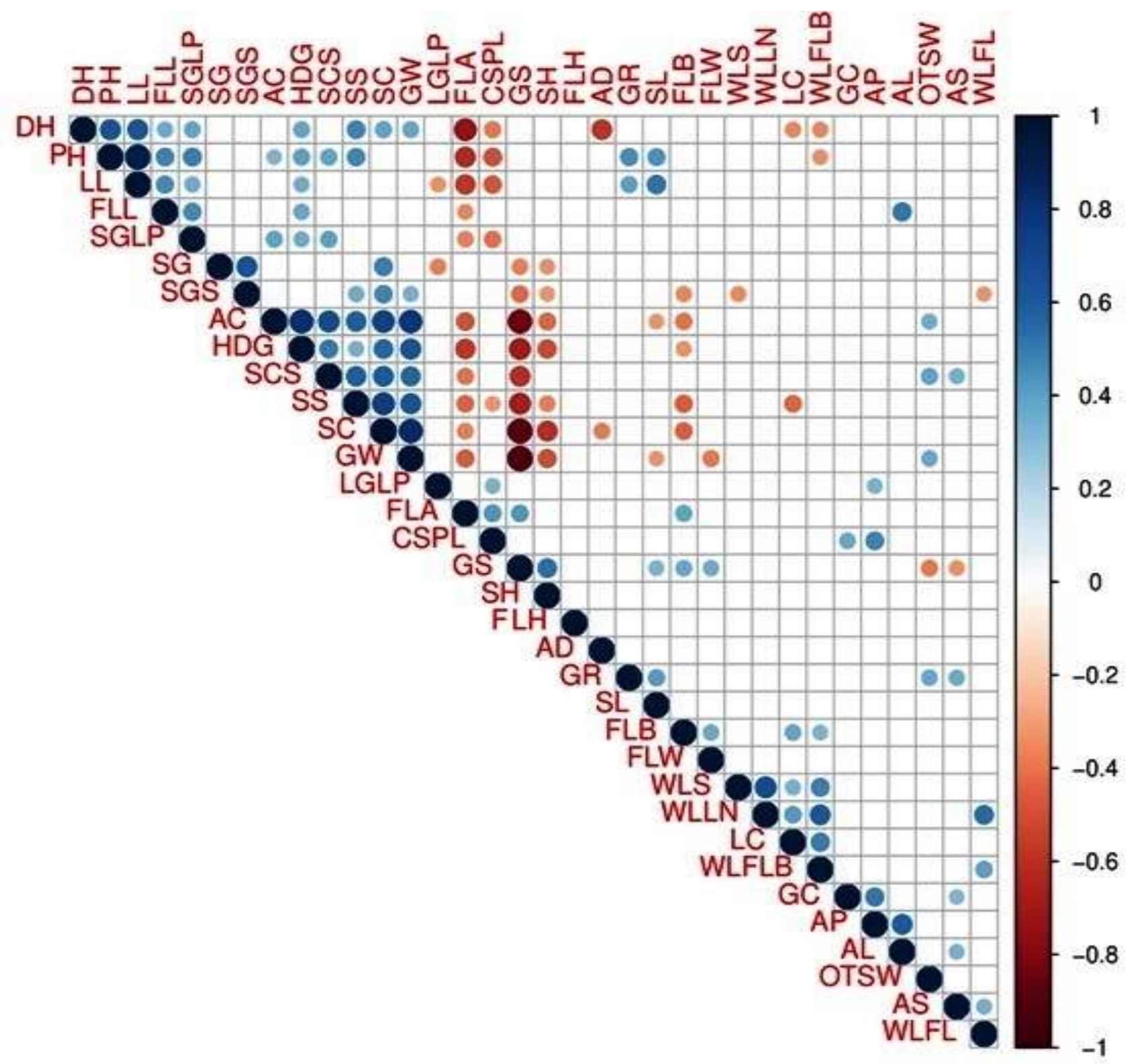

Fig. 1. The significant correlation analysis of $\mathbf{r}$ between different morpho-agronomic traits of wheat accessions. The blue and red circles indicate positive and negative correlation, respectively. The circles size is relative to correlation values. 


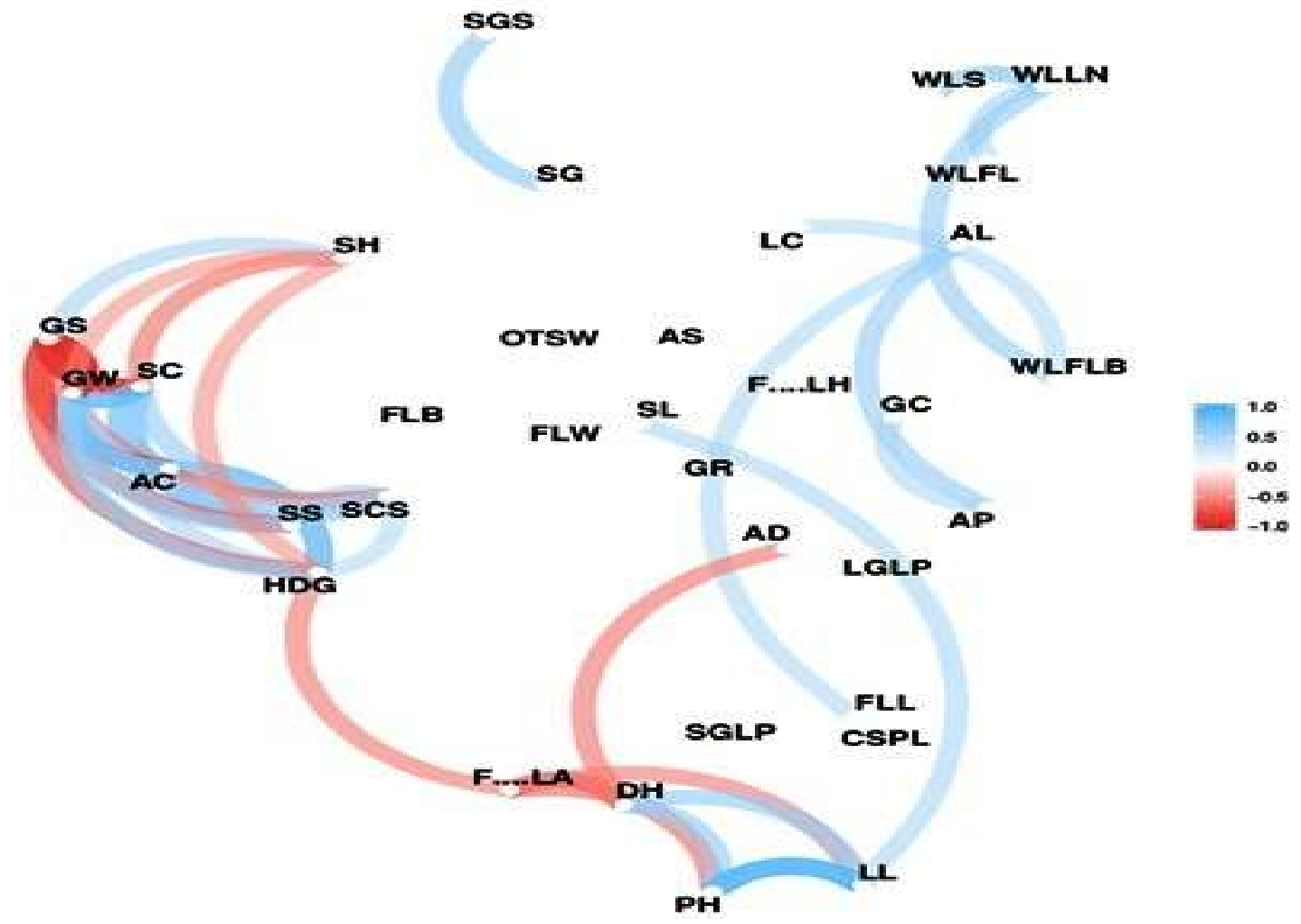

Fig. 2. The correlation network between different wheat morpho-agronomic traits, where of $r>0.5$. The blue and red links indicates positive and negative correlation, respectively.

\section{ISSR15}

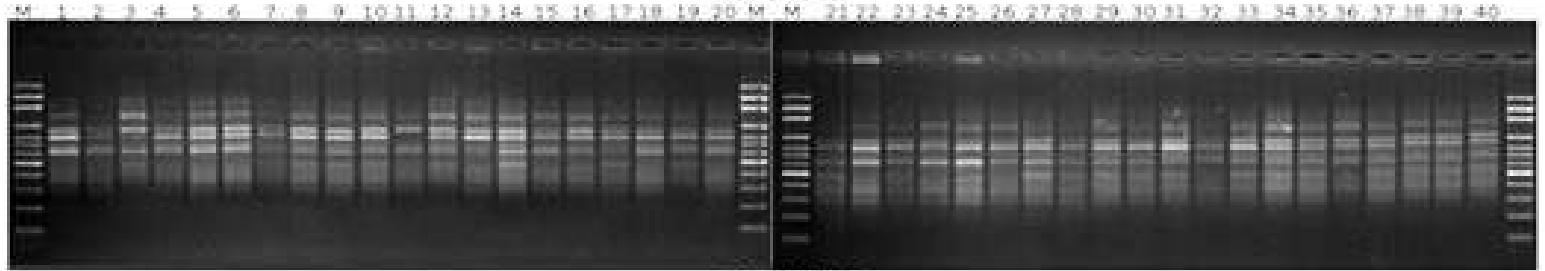

SCoT16

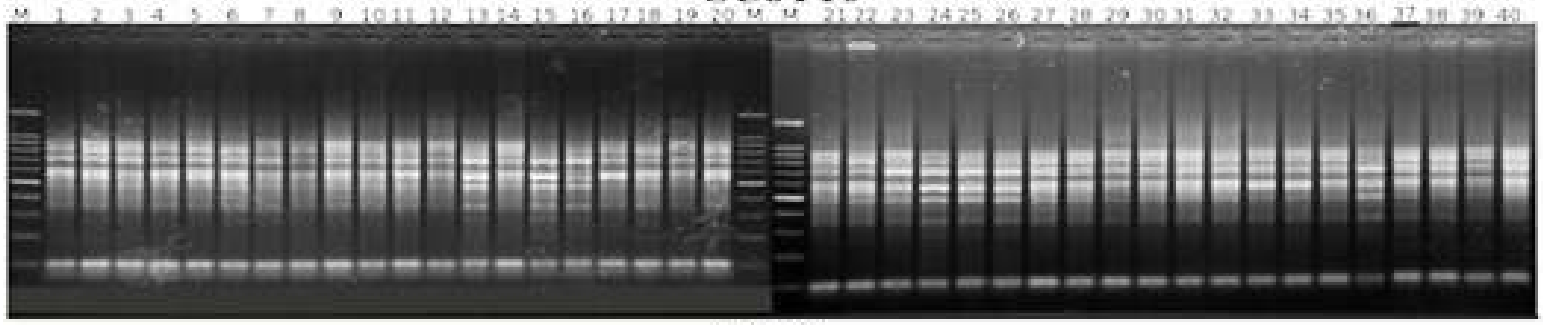

SSR2

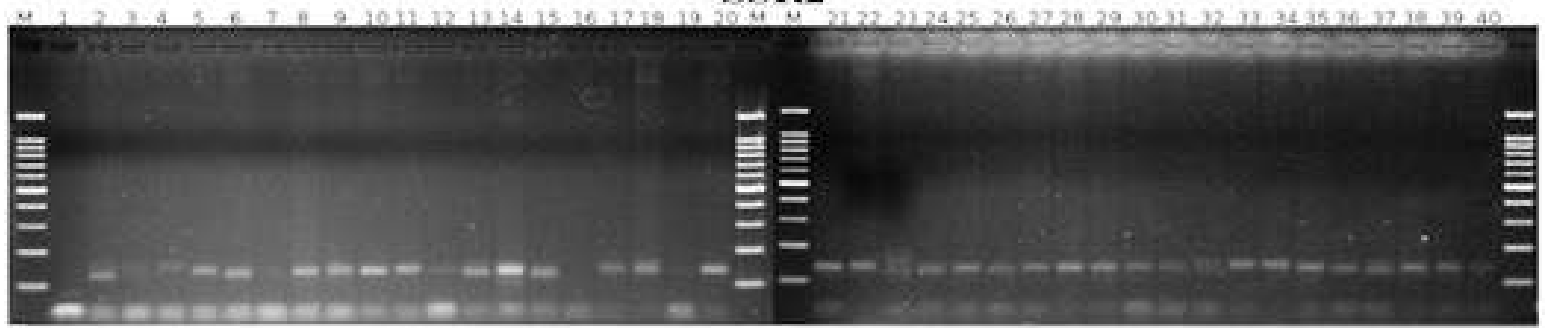

Fig. 3. The gel electrophoresis for the 40 wheat accessions analyzed using three different PCR primers. 


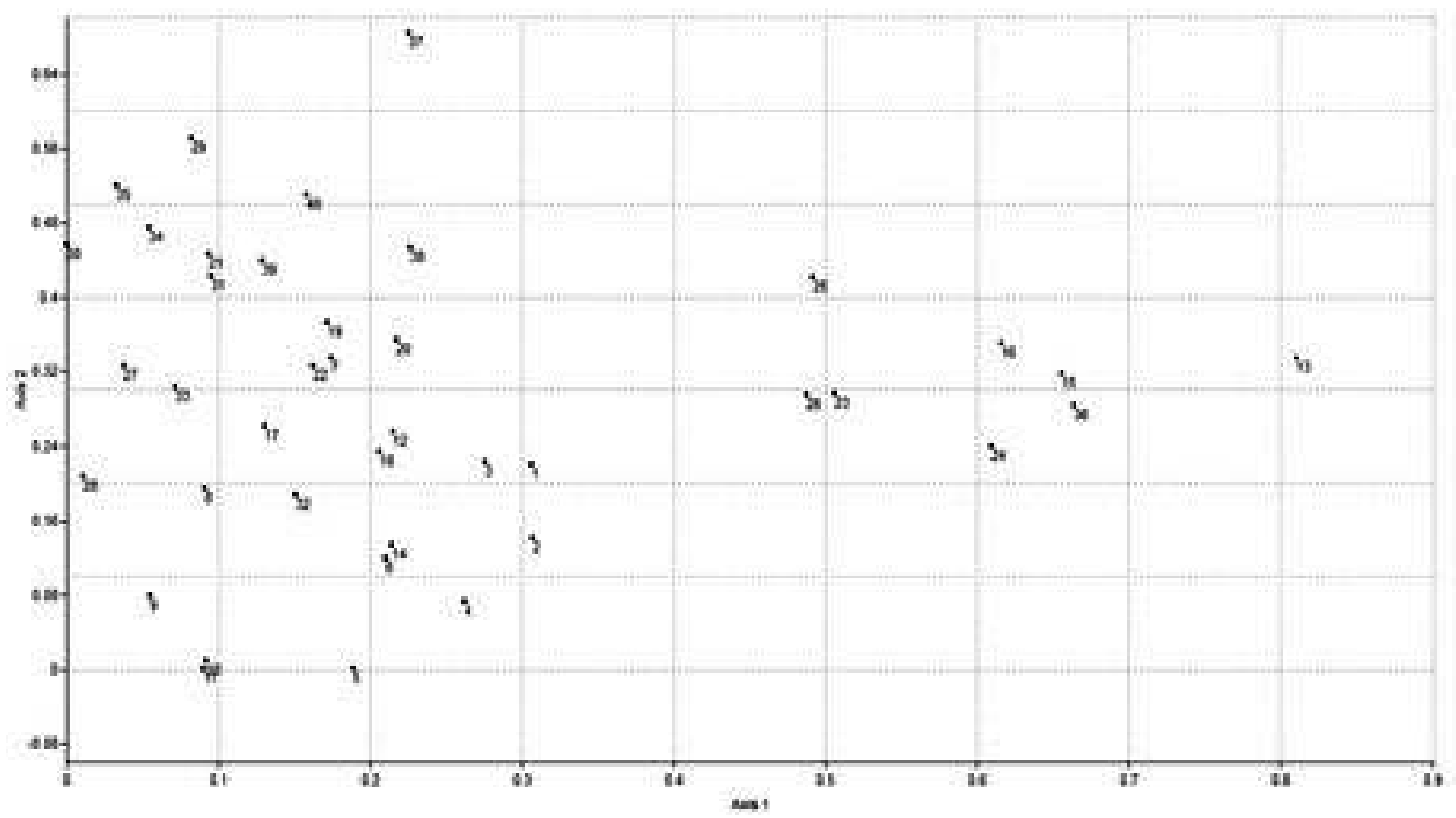

Fig. 4. The DCA-based population structure analysis for the 40 wheat accessions used in this study.

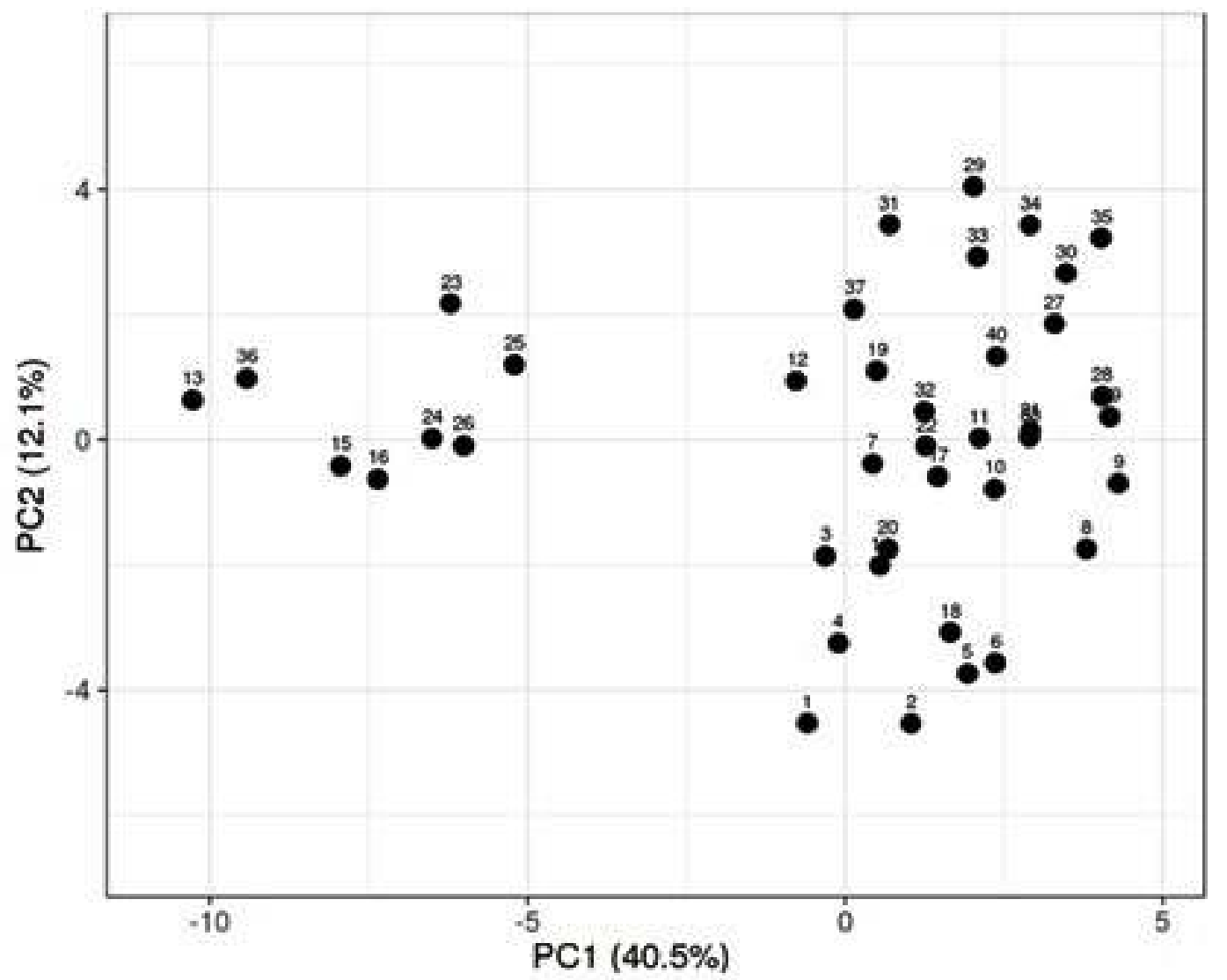

Fig. 5. The PCA-based population structure analysis for the 40 wheat accessions used in this study. 


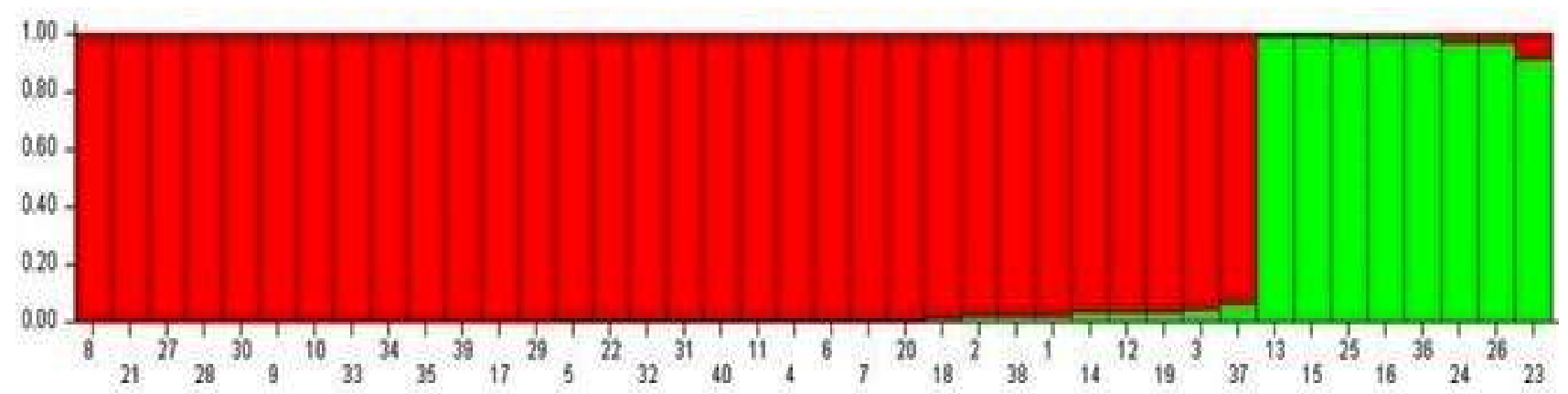

Fig. 6. The alleles frequencies-based population structure analysis using STRUCTURE software for the 40 wheat accessions used in this study.

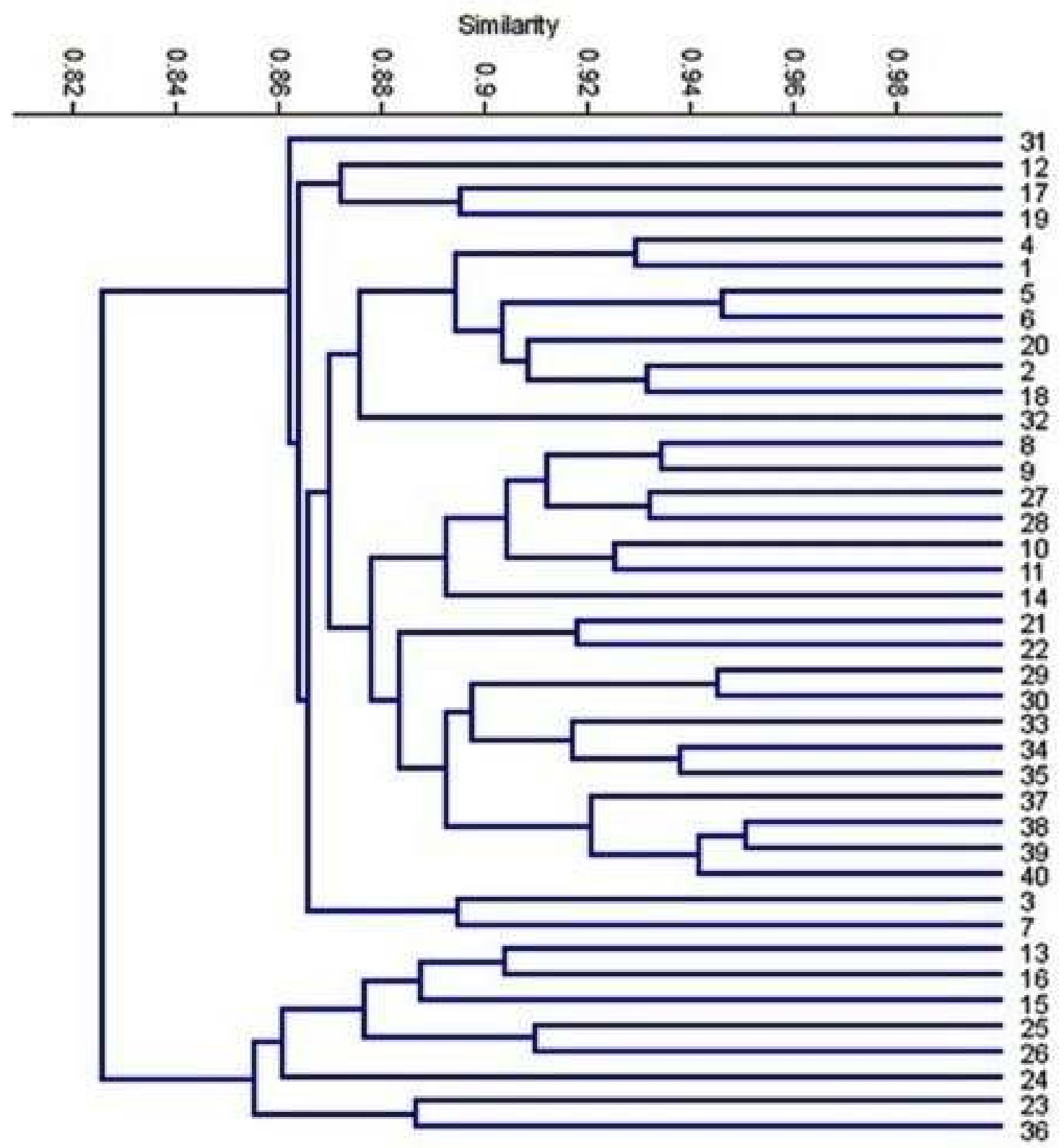

Fig. 7. The phylogenetic tree of the 40 wheat accessions constructed using binary data retrieved from ISSR, SSR and SCoT marker assays. 


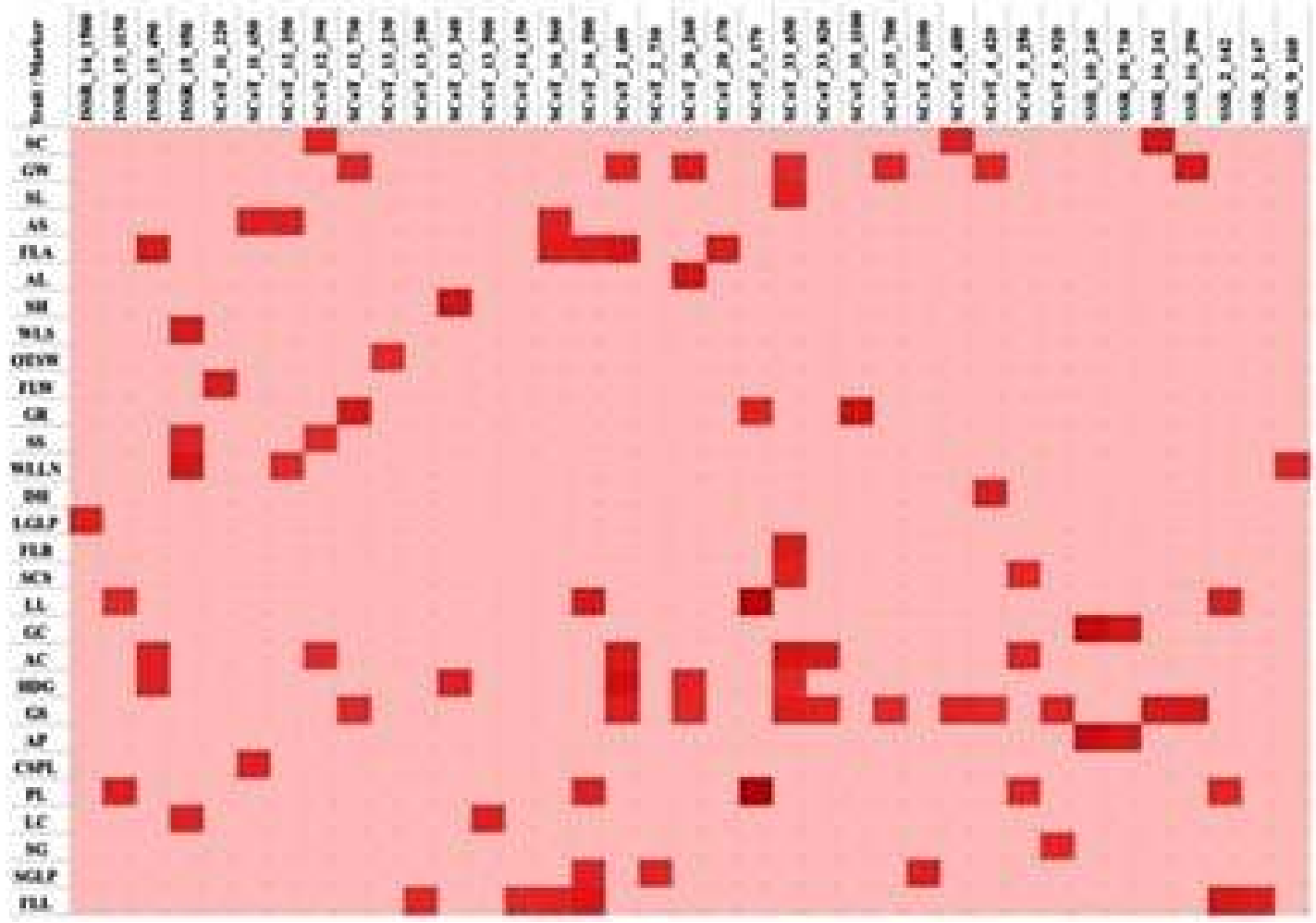

Fig. 8. The multiple traits controlling markers, the red color concentration is relative to the p-value score (highest scores have darker red colors).

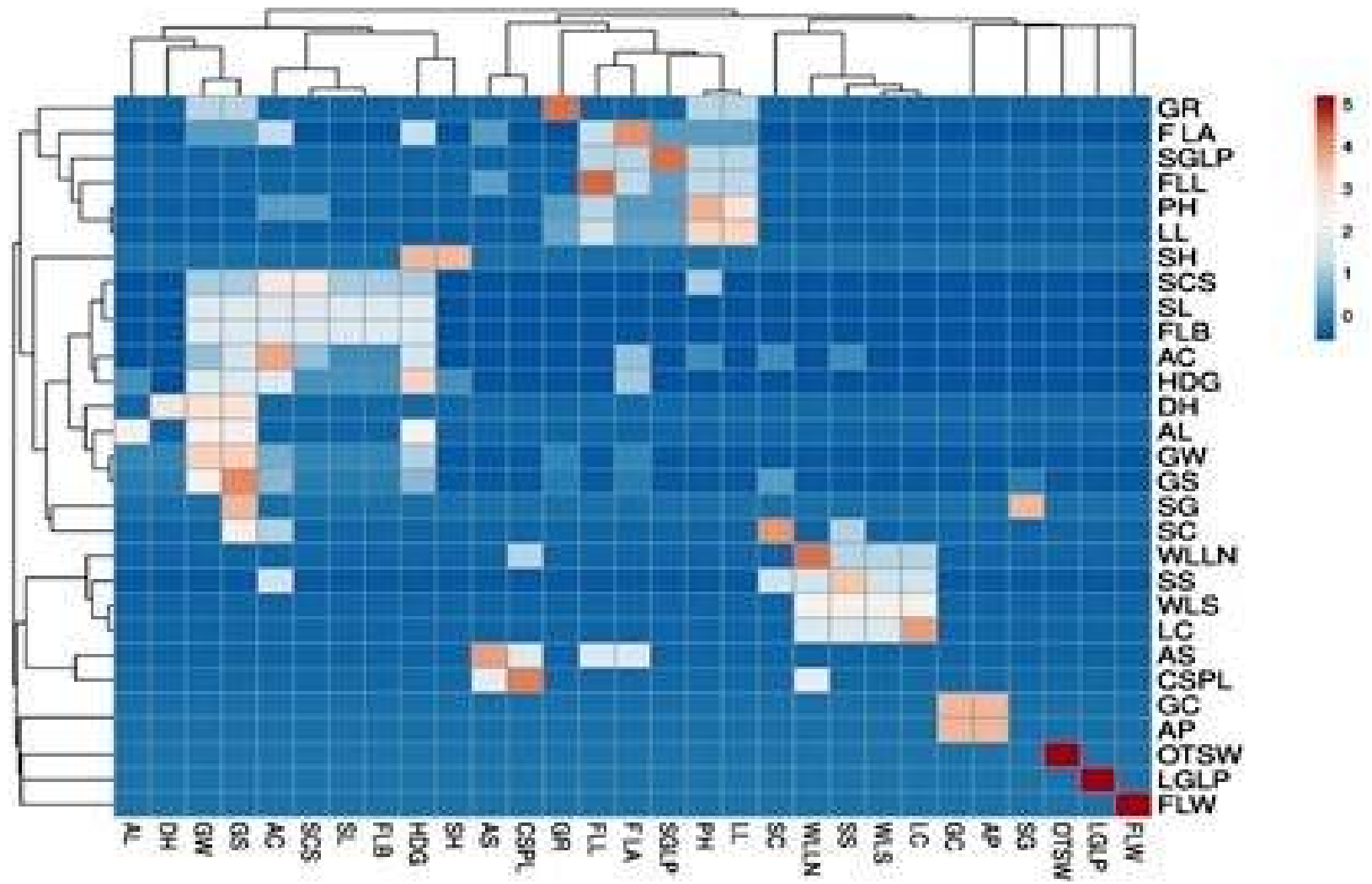

Fig. 9. Heatmap for significant shared markers between traits, the blue and red color scale (left side) is relative to the number of shared markers. 
Conclusion: Statistical associations between different morpho-agronomic traits of some wheat genotypes grown in Saudi Arabia were studied in order to estimate the hidden correlation network and to detect few traitassociated markers which could be used in markerassisted selection. The studies on associations between traits in wheat revealed a robust correlation between $\mathrm{DH}$ and FLA, PH and LL, SCS and AC, and SC and GS. These correlations could be used to study the impact of traits on wheat yield and the effect of the genotypic variation in wheat. Moreover, by using different molecular markers, several markers associated with different wheat traits were identified. These markers could be used in national wheat breeding programs for developing and selecting the most adaptive and productive genotypes.

Acknowledgements: We are sincerely thank the Employees in Plant Gene bank in National Agriculture \& Animal Resources Research Center, Ministry of Environment Water \& Agriculture in Saudi Arabia who worked on sample collection and their kind cooperation.

\section{REFERENCES}

Abdein, M. A., D. Abd El-Moneiem, I.M. Sahar, S. Taha, Widad, S. M. Al-Juhani, Wa. Sal, E. Mohamed. (2018) Molecular characterization and genetic relationships among some tomato genotypes as revealed by ISSR and SCoT markers. Egypt. J. Genet. Cytol.. 47:1-8.

Ahla, A. Awad., S. D. Ibrahim, S. S. Adawy, and M. A. Omar. (2019) Development of AFLP, ISSR and RAPD markers for high yield related traits in Jojoba, Egypt. J. Genet. Cytol.. 47(2): 279-294

Alireza, Etminan., Alireza. Pour-Aboughadareh., Reza. Mohammadi., Amin. Ahmadi-Rad., Afsaneh. Noori., Zahra. Mahdavian., Zahra. Moradi. (2016) Applicability of start codon targeted (SCoT) and inter-simple sequence repeat (ISSR) markers for genetic diversity analysis in durum wheat genotypes. Biotechnology and Biotechnological Equipment. 30(6): 1075-1081.

Alsamman, A. M., S. D. Ibrahim, and A. Hamwieh, (2019) KASPspoon: an in vitro and in silico PCR analysis tool for high-throughput SNP genotyping. Bioinformatics. 35(17): 3187-3190.

Alsamman, M. Alsamman., S. S. Adawy, S. D. Ibrahim, B. A. Hussein, and H. A. Hussein. (2017) Selective Amplification of Start codon Polymorphic Loci (SASPL): a new PCR-based molecular marker in olive. Plant Omics. 10(2): 64-75.

Collard, B. C. Y., and D. J. Mackill. (2009) Start codon targeted (SCoT) polymorphism: a simple, novel DNA marker technique for generating genetargeted markers in plants. Plant molecular biology reporter. 27(1): 86-93.

Curtis, B. C ., S. Rajaram, Gómez. and H. Macpherson. (2002) Bread wheat: improvement and production. Food and Agriculture Organization of the United Nations (FAO).

Danail, Kahrizi., Maniee. Mahdi, Mohammadi. Reza, and Cheghamirza. Kianoosh. (2010) Estimation of genetic parameters related to morpho-agronomic traits of Durum Wheat (Triticum turgidum var. durum). Biharean Biologist. University of Oradea Publishing House. 4(2): 93-97.

Earl, D. A., and B. M. von Holdt (2012) STRUCTURE HARVESTER: a website and program for visualizing STRUCTURE output and implementing the Evanno method. Conservation Genetics Resources. 4(2): 359-361.

Elakhdar, A., A. M. El-Sattar, K. Amer, A. Rady, and T. Kumamaru, (2016) Population structure and marker--trait association of salt tolerance in barley (Hordeum vulgare L.) Comptes rendus biologies. 339(11-12): 454-461.

Fernandez, M., A. Figueiras, and C. Benito. (2002) The use of ISSR and RAPD markers for detecting DNA polymorphism, genotype identification and genetic diversity among barley cultivars with known origin. Theoretical and Applied Genetics. 104(5): 845-851.

Figueroa, M., K. E. Hammond-Kosack, and P. S. Solomon. (2018) A review of wheat diseases - a field perspective. Molecular plant pathology. 19(6): 1523-1536.

Fischer, R. A. (2011) Wheat physiology: a review of recent developments. Crop and Pasture Science. 62(2): 95-114.

Gomes, S.O., R.F.M. Mendes, R.L.F. Gomes, M.E.C. Veloso, N.H.C. Arriel, D.M.P. Azevedo G.M.C. Carvalho, and P.S.C. Lima. (2018) Assessment of the genetic diversity and population structure of Jatropha curcas accessions in Brazil using ISSR markers. Embrapa Meio-Norte-Artigo em periódico indexado (ALICE), Genetics and Molecular Research. 17: GMR18208DOI: $10.4238 / \mathrm{gmr} 18208$.

Gondo, T., S. Sato, K. Okumura, S. Tabata, R. Akashi, S. and Isobe. (2007) Quantitative trait locus analysis of multiple agronomic traits in the model legume Lotus japonicus. Genome. 50(7): 627-637.

Habib, P. T., A. M. Alsamman, and A. Hamwieh. (2019) BioAnalyzer: Bioinformatic Software of Routinely Used Tools for Analysis of Genomic Data. Biotechnology. 10: 33-41.

Harrell, Jr., F. E. Harrell, M. F. E. Jr. (2019) Package "Hmisc", CRAN20.18: 235-236.

Heikrujam, M., J. Kumar, and V. Agrawal. (2015) Genetic diversity analysis among male and 
female Jojoba genotypes employing gene targeted molecular markers, start codon targeted (SCoT) polymorphism and CAAT box-derived polymorphism (CBDP) markers. Meta gene. 5: 90-97.

Hill, M. O., and H. G., Gauch (1980) Detrended correspondence analysis: an improved ordination technique. in Classification and ordination, Springer. 47-58.

Lee, C., A. Abdool, and C.-H. Huang. (2009) PCA-based population structure inference with generic clustering algorithms. BMC bioinformatics. 10(1): S73.

Liu, K., and S. V . Muse. (2005) Power Marker: an integrated analysis environment for genetic marker analysis. Bioinformatics. 21(9): 21282129.

Lowe, W. L., and T. E. Reddy. (2015) Genomic approaches for understanding the genetics of complex disease. Genome research. 25(10): 1432-1441.

Metsalu, T., and J. Vilo. (2015) ClustVis: a web tool for visualizing clustering of multivariate data using Principal Component Analysis and heatmap. Nucleic acids research. 43(W1): W566-W570.

Muhammad, Farooq., Helen. Bramley, Jairo. A. Palta. Kadambot, H.M. Siddique. (2011) Heat stress in wheat during reproductive and grain-filling phases. Critical Reviews in Plant Sciences. 30(6): 491-507.

Munir, Ahmad., Armghan. Shahzad, Muhammad. Iqbal, Muhammad. Asif, Arvind. H. Hirani. and (2013) Morphological and molecular genetic variation in wheat for salinity tolerance at germination and early seedling stage. Australian J. Crop Science. 7(1): 66-74.

Munshi, Abdulla., and Osman. H. Gamal. (2010) Investigation on molecular phylogeny of some date palm (Phoenix dactylifra L.) cultivars by protein, RAPD and ISSR markers in Saudi Arabia. AJCS . 4(1):23-28

Pritchard, J. K., M. Stephens, and P. , Donnelly. (2000) Inference of population structure using multilocus genotype data. Genetics. 155(2): 945-959.

Qi, L.L., M.O. Pumphrey, B. Friebe, P.D. Chen, and B.S. Gill. (2008) Molecular cytogenetic characterization of alien introgressions with gene Fhb3 for resistance to Fusarium head blight disease of wheat. Theoretical and Applied Genetics. 117(7): 1155-1166.

Quarrie, S. A., D. Dodig, S., Pekiç, J. Kirby, and B. Kobiljski . (2003) Prospects for marker-assisted selection of improved drought responses in wheat. Bulgarian J. Plant Physiology. 28: 83-95.

Rajesh, M. K., A. A. Sabana, K. E. Rachana, Shafeeq. Rahman., B. A. Jerard, and Anitha. Karun . (2015) Genetic relationship and diversity among coconut (Cocos nucifera L.) accessions revealed through SCoT analysis. 3 Biotech. 5(6): 999 1006.

Saima, Mir., Arain. Mahboob., Ali. Sial., Karim. Dino, Jamali. Khalil, and Ahmed. Laghari. (2018) Grain yield performance, correlation, and cluster analysis in elite bread wheat (Triticum aestivum L.) lines. Acta Agrobotanica. 71(4): 1747-1756

Ibrahim , Shafik., S. S. Adawy, M. A. M. Atia, A. M. Alsamman, and M. M. Mokhtar. (2016) Genetic diversity, variety identification and gene detection in some Egyptian grape varieties by SSR and SCoT markers. Plant Omics. 9(5): 311 318.

Shaimaa, M., A.M. Ahmed, M.H. Alsamman, M.A. Mubarak, M.A. Badawy. Kord, O.A. Momtaz, and A. Hamwieh. (2019) Dowsing for salinity tolerance related genes in chickpea through genome wide association and in silico PCR analysis. bioRxiv. Cold Spring Harbor Laboratory. p. 519744.

Skoracka, A., B. G. Rector, and G. L., Hein. (2018) The interface between wheat and the wheat curl mite, Aceria tosichella, the primary vector of globally important viral diseases. Frontiers in plant science. Frontiers Media SA, 9.

Taiyun, Wei ., Viliam. Simko., and Maintainer. Taiyun. Wei . (2017) Package "corrplot. Statistician. 56: 316-324

Westendorf, M. L. (2000) Food waste as animal feed: an introduction. Food waste to animal feed. Wiley Online Library. 3-16.

Yong-Cui, Hou., Ze-Hong. Yan, Yu-Ming. Wei, YouLiang, Zheng. (2005) Genetic diversity in barley from west China based on RAPD and ISSR analysis. Barley Genetics Newsletter. Citeseer. 35(1): 9-22. 